\title{
Charged Higgs Prospects with CMS
}

\section{Ritva Kinnunen*i}

Helsinki Institute of Physics

E-mail: ritva.kinnunen@cern.ch

Prospects for Charged Higgs Discovery at Colliders

16-19 September 2008

Uppsala, Sweden

\footnotetext{
*Speaker.

${ }^{\dagger}$ On behalf of the CMS Collaboration
} 


\section{Introduction}

The Minimal Supersymmetric Standard Model (MSSM) [1],[2],[3] predicts existence of two charged Higgs bosons. Direct searches at LEP have yield a lower bound of $\mathrm{m}_{\mathrm{H}^{ \pm}}>125 \mathrm{GeV} / \mathrm{c}^{2}$ for the mass of the charged Higgs bosons in the CP-conserving MSSM [4]. At the LHC, the light charged Higgs bosons with $\mathrm{m}_{\mathrm{H}^{ \pm}}<\mathrm{m}_{\text {top }}$ can be produced in the $\mathrm{t}_{\mathrm{t}}$ events through the $\mathrm{t} \rightarrow \mathrm{bH}^{ \pm}$ decay. Production of the heavy charged Higgs bosons proceeds mainly through the associated production processes $\mathrm{gb} \rightarrow \mathrm{tH}^{ \pm}$and $\mathrm{gg} \rightarrow \mathrm{tbH}^{ \pm}$. The light charged Higgs bosons decay almost exclusively with the $\mathrm{H}^{ \pm} \rightarrow \tau^{ \pm} v_{\tau}$ decay mode. For the heavy charged Higgs bosons the $\mathrm{H}^{ \pm} \rightarrow \mathrm{tb}$ decay mode dominates but the sub-dominant decay mode $\mathrm{H}^{ \pm} \rightarrow \tau^{ \pm} v_{\tau}$ is more promising as a discovery channel [5]. The advantages of the $\mathrm{H}^{ \pm} \rightarrow \tau^{ \pm} v_{\tau}$ decay mode are the possibility to exploit the $\tau$ helicity correlations and the possibility to reconstruct the transverse mass from the identified $\tau$ jet and from the measured missing transverse energy (MET) from the $\mathrm{H}^{ \pm}$produced in association with a fully hadronic final state.

In the following searches for light charged Higgs bosons are discussed in Section 3. Searches of the heavy charged Higgs bosons with the $\mathrm{H}^{ \pm} \rightarrow$ tb decay channel are presented in Section 4 and with the $\mathrm{H}^{ \pm} \rightarrow \tau^{ \pm} v_{\tau}$ decay channel in Section 5. The results are based on full simulation and reconstruction of the CMS detector. The discovery regions are calculated for the following MSSM parameters: $\mathrm{M}_{2}=200 \mathrm{GeV} / c^{2}, \mu=200 \mathrm{GeV} / c^{2}, \mathrm{M}_{3}=800 \mathrm{GeV} / c^{2}, \mathrm{M}_{\mathrm{SUSY}}=1 \mathrm{TeV} / c^{2}$. The maximal mixing scenario fixing $\mathrm{X}_{\mathrm{t}}\left(\mathrm{X}_{\mathrm{t}}=\mathrm{A}_{\mathrm{t}}-\mu \cot \beta\right)$ to $2 \mathrm{TeV} / c^{2}$ is assumed. For the $\mathrm{H}^{ \pm} \rightarrow$ $\tau^{ \pm} v_{\tau}$ decays channel new studies are in progress aiming for background measurements with the early LHC data. Discussion and some qualitative results on these studies are included in Section 6. An important feature for the $\mathrm{H}^{ \pm} \rightarrow \tau^{ \pm} v_{\tau}$ decay channels is the possibility to exploit the $\tau$ polarisation to suppress the backgrounds with $\mathrm{W}^{ \pm} \rightarrow \tau^{ \pm} v_{\tau}$ decays. The principle of this method is first discussed in Section 2.

\subsubsection{Helicity correlations}

The polarisation states for the $\tau^{+}$from $\mathrm{H}^{+} \rightarrow \tau^{+} v_{\tau}$ and from $\mathrm{W}^{+} \rightarrow \tau^{+} v_{\tau}$ are opposite due to the spin-parity properties of the decaying particle. The angular distribution of a pion from the $\tau^{ \pm} \rightarrow \pi^{ \pm} v$ decay in the $\mathrm{CM}$ frame has the form $\left(1+\mathrm{P}_{\tau} \cos \theta\right)$, which leads to more energetic charged pions in the laboratory frame for the signal $\left(\mathrm{P}_{\tau}=1\right)$ than for the background $\left(\mathrm{P}_{\tau}=-1\right)$ [5],[6]. The $\tau^{ \pm} \rightarrow \pi^{ \pm} v_{\tau}$ decay channel presents $12.5 \%$ of the hadronic decay modes. The charged pion is more energetic also in the $\tau$ decays to vector mesons and subsequent decays to one charged pion in the longitudinal polarisation states of the vector meson, $\tau^{ \pm} \rightarrow \rho_{\mathrm{L}}^{ \pm} v_{\tau} \rightarrow \pi^{ \pm} \pi^{\circ} v_{\tau}(26 \%)$ and $\tau^{ \pm} \rightarrow \mathrm{a}_{1 \mathrm{~L}}^{ \pm} \nu_{\tau} \rightarrow \pi^{ \pm} \pi^{\circ} \pi^{\circ} v_{\tau}(7.5 \%)$. For the transverse polarisation states of the vector meson the situation is opposite with more energetic pions from the background. The small contributions from $\mathrm{K}^{*}$ and $\mathrm{K}$ in the $\tau$ decays lead to similar effects. The helicity correlations can be expressed as a function of the $\tau$-jet momentum fraction carried by the charged pion $\mathrm{R}_{\tau}=\mathrm{p}_{\pi} / \mathrm{p}_{\tau}$ jet. As is shown in Refs. [5],[6] the $\tau^{ \pm} \rightarrow \pi^{ \pm} v_{\tau}$ decay leads to a $\delta$-function at $\mathrm{R}_{\tau}=1$, the $\rho_{\mathrm{L}}^{ \pm} v_{\tau} \rightarrow \pi^{ \pm} \pi^{\circ} v_{\tau}$ has contributions at $\mathrm{R}_{\tau} \sim 1$ and $\mathrm{R}_{\tau} \sim 0, \rho_{\mathrm{T}}^{ \pm} v_{\tau} \rightarrow \pi^{ \pm} \pi^{\circ} v_{\tau}$ and $\mathrm{a}_{1 \mathrm{~T}}^{ \pm} v_{\tau} \rightarrow \pi^{ \pm} \pi^{\circ} \pi^{\circ} v_{\tau}$ have largest contributions around $\mathrm{R}_{\tau} \sim 0.5$ while $\mathrm{a}_{1 \mathrm{~L}}^{ \pm} \nu_{\tau} \rightarrow \pi^{ \pm} \pi^{\circ} \pi^{\circ} v_{\tau}$ peaks at $\mathrm{R}_{\tau} \sim 0$. In the following studies the $\tau$ decays are generated with TAUOLA [7] to include the polarisation effects. 


\section{Searches for the light charged MSSM Higgs bosons with $\mathrm{H}^{+} \rightarrow \tau^{+} v_{\tau}$}

For the light charged Higgs bosons $\left(\mathrm{m}_{\mathrm{H}^{ \pm}}<\mathrm{m}_{\mathrm{top}}\right)$ the topology where one of the top quarks decays leptonically to trigger the event with the single lepton triggers was studied in CMS [8],[9].

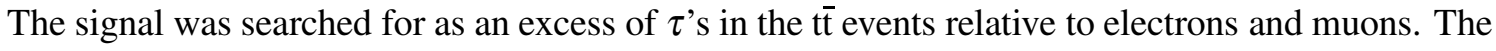
background consist of $\mathrm{t}_{\mathrm{t}}$ events with at least one lepton (e or $\mu$ ) and $\tau$-jets or jets which could fake $\tau$-jets, $\mathrm{W}^{ \pm}+3$ jet events and single top (Wt) events. The $\mathrm{t} \overline{\mathrm{t}}, \mathrm{gb} \rightarrow \mathrm{tH}^{ \pm}$and $\mathrm{gg} \rightarrow \mathrm{t} \overline{\mathrm{b}} \mathrm{H}^{ \pm}$processes were generated with PYTHIA [10]. The Wt background was generated with TOPREX [11] and the $\mathrm{W}+3 \mathrm{j}$ background with MadGraph [12]. The production cross sections for $\mathrm{t} \overline{\mathrm{t}}$ and $\mathrm{Wt}$ processes were normalised to the NLO cross sections. Other background processes such as Wbb, Zbb with $\mathrm{W} \rightarrow \ell v(\ell=\mathrm{e}, \mu)$ and $\mathrm{Z} \rightarrow \mathrm{ee}$, or $\tau \tau$ were investigated and were found to be negligible.

The hadronic $\tau$ decays were identified with a cone algorithm on reconstructed hadronic jets with $\mathrm{E}_{\mathrm{T}}>40 \mathrm{GeV}$. A deposition of $\mathrm{E}_{\mathrm{T}}>2 \mathrm{GeV}$ as required in at least one Hadron Calorimeter (HCAL) tower in the jet to suppress the electron contamination. One or three tracks were required in the signal cone of $\Delta \mathrm{R}=0.07$. Isolation was performed in the tracker and in the electromagnetic calorimeter (ECAL) with an isolation cone of $\Delta \mathrm{R}=0.4$ around this signal cone. For the ECAL isolation the signal cone was enlarged to $\Delta \mathrm{R}=0.13$. The leading track in the $\tau$-jet candidate was required to carry at least $80 \%$ of the energy of the jet to exploit the $\tau$ helicity correlations in the $\mathrm{H}^{ \pm} \rightarrow \tau \nu_{\tau}$ and the $\mathrm{W}^{ \pm} \rightarrow \tau \nu_{\tau}$ decays. The requirement $\mathrm{Q}($ lepton $)+\mathrm{Q}(\tau$ jet $)=0$ was set on the charges of the $\tau$ jet and the triggered lepton $\left(\mathrm{p}_{\mathrm{T}}>20 \mathrm{GeV}\right)$. At least three hadronic jets with $\mathrm{E}_{\mathrm{T}}>40 \mathrm{GeV}, \mathrm{MET}>70 \mathrm{GeV}$ and one tagged $\mathrm{b}$ jet were required in the event.

The systematic uncertainties of the signal significance were estimated on the basis of the experimental selection uncertainty of the background events and the theoretical cross section calculation uncertainty of the $t \bar{t}$ and single top background. A method to measure the $\mathrm{W}+3$ jet background from the real data was developed. A signal-free background area was defined demanding three hadronic jets and excluding all b-jets and $\tau$ jets in the lepton + multi-jet events. The uncertainty of the measurement was estimated by propagating the contribution of events counted in the background area to the signal area and cancelling the common selection cut uncertainties. Figure 1 shows $5 \sigma$-discovery potential of CMS for light charged MSSM Higgs bosons with the $\mathrm{H}^{ \pm} \rightarrow \tau \nu_{\tau}$ decay channel as a function of $\mathrm{m}_{\mathrm{A}}$ and $\tan \beta$ for $30 \mathrm{fb}^{-1}$ in the maximal mixing scenario with $\mu=200 \mathrm{GeV} / c^{2}$.

\section{Searches for charged MSSM Higgs bosons with $\mathrm{H}^{ \pm} \rightarrow$ tb}

The two production channels $\mathrm{gb} \rightarrow \mathrm{tH}^{ \pm}$and $\mathrm{gg} \rightarrow \mathrm{tbH}^{ \pm}$were considered in the search for charged Higgs bosons in the $\mathrm{H}^{ \pm} \rightarrow$ tb decay channel [13],[9]:

$$
\begin{gathered}
\mathrm{gb} \rightarrow \mathrm{tH}^{ \pm} \rightarrow \mathrm{ttb} \rightarrow \mathrm{W}^{+} \mathrm{W}^{-} \mathrm{bbb} \rightarrow \mathrm{qq}^{\prime} \mu v_{\mu} \mathrm{bbb}, \\
\mathrm{gg} \rightarrow \mathrm{tH}^{ \pm} \mathrm{b} \rightarrow \mathrm{ttbb} \rightarrow \mathrm{W}^{+} \mathrm{W}^{-} \mathrm{bbbb} \rightarrow \mathrm{qq}^{\prime} \mu v_{\mu} \mathrm{bbbb} .
\end{gathered}
$$

For both processes, the cross section was rescaled to the NLO result for the $\mathrm{pp} \rightarrow \mathrm{tH}^{ \pm} \mathrm{X}$ channel [14]. The events were assumed to be triggered with a single muon trigger. The final state (3.1) was studied with triple $b$ tagging while four tagged $b$ jets were required for the the final state (3.2). The generation of both production processes was performed with PYTHIA [10]. For the process 

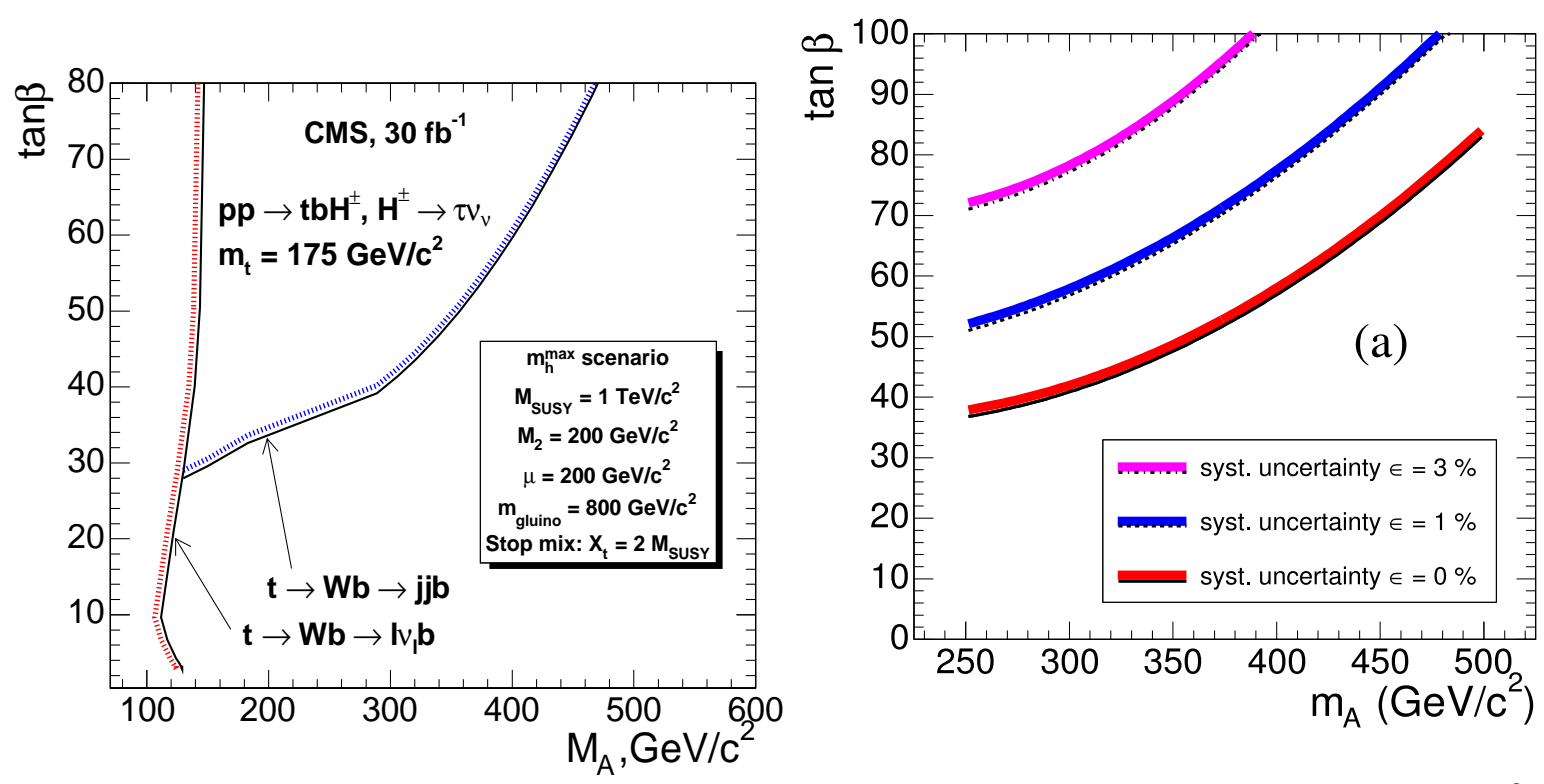

Figure 1: The $5 \sigma$-discovery region in the $\mathrm{m}_{\mathrm{A}}, \tan \beta$ plane for charged Higgs bosons with the $\mathrm{H}^{ \pm} \rightarrow$ $\tau v_{\tau}$ decay channel with an integrated luminosity of $30 \mathrm{fb}^{-1}$.

Figure 2: The $5 \sigma$-discovery region in the $\mathrm{m}_{\mathrm{A}}, \tan \beta$ plane for charged Higgs bosons with the $\mathrm{H}^{ \pm} \rightarrow$ tb decay channel for $30 \mathrm{fb}^{-1}$ assuming precise knowledge of the background (solid line) and a systematic uncertainties of $1 \%$ (dashed line) and 3\% (dash-dotted line).

(3.1) the main backgrounds are due to $\mathrm{pp} \rightarrow \mathrm{t} \overline{\mathrm{tb}}$ and $\mathrm{pp} \rightarrow \mathrm{t} \overline{\mathrm{t}}+$ jet production, where in the latter the extra jet is misidentified as a $b$ jet. The event simulation was performed with MadGraph [12]. The background for the process (3.2) consists of the irreducible $\mathrm{pp} \rightarrow \mathrm{t} \overline{\mathrm{t}} \mathrm{b} \overline{\mathrm{b}}$ and the reducible $\mathrm{pp} \rightarrow \mathrm{t} \overline{\mathrm{t} j \mathrm{j}}$ process, where in the latter two jets are misidentified as $b$ jets. Both these backgrounds were generated with the COMPHEP program [15].

Events passing the single muon trigger were required to have one muon with $\mathrm{p}_{\mathrm{T}}>20 \mathrm{GeV} / \mathrm{c}$ and $|\eta|<2.5$. For the process (3.1) at least five jets with $\mathrm{E}_{\mathrm{T}}>25 \mathrm{GeV}$ and $|\eta|<2.5$ and at least three of these jets tagged as $b$ jets with a secondary vertex-based algorithm [16] were required. For the process (3.2) one more jet and one more $b$ jet was required. The best jet association was selected with a likelihood ratio technique, which combines information from kinematic properties of the extra jets, b-tagging of all jets and the result of a kinematic fit on the $t \bar{t}$ system, imposing both $\mathrm{W}^{ \pm}$and top mass constraints. Starting from the chosen jet association the Higgs boson mass was reconstructed. Due to the large combinatorial background, the mass information was not used further on in the analysis. To suppress the large $t \bar{t}+$ jets background, observables were identified that have different properties for signal and background events. These observables were combined into an overall discriminator, in which a cut was set to separate the signal and background contributions. Due to the large background remaining in both final states the effect of systematic uncertainties on the knowledge of the background is important. Figure 2 shows the discovery contours for the final states (3.1) with triple $\mathrm{b}$ tagging, when supposing perfect knowledge of the background cross section $(\varepsilon=0)$, a $1 \%$ uncertainty $(\varepsilon=0.01)$, and a $3 \%$ uncertainty $(\varepsilon=0.03)$. No visibility can be 
obtained for this channel in the MSSM parameter space during the low luminosity phase of LHC. For the final state (3.2) with four tagged $b$ jets the discovery region is even more limited [13].

\section{Searches for charged MSSM Higgs bosons with $\mathrm{H}^{ \pm} \rightarrow \tau v$}

The $\mathrm{H}^{ \pm} \rightarrow \tau \nu$ channel for was studied in Refs. [9],[17] for $\mathrm{m}_{\mathrm{H}^{ \pm}}>\mathrm{m}_{\mathrm{top}}$. The process $\mathrm{gg} \rightarrow$ $\mathrm{tbH}^{ \pm}$was used for the signal production normalising the cross sections to the NLO results of Ref. [14]. The signal, $t \bar{t}$ and the QCD multi-jet events were generated with PYTHIA, the W+3jet events with MadGraph and the Wt events with TOPREX. A single $\tau$-jet $\left(\mathrm{E}_{\mathrm{T}}>96 \mathrm{GeV}\right)$ trigger combined with a MET cut $(\mathrm{MET}>68 \mathrm{GeV})$ at the HLT was assumed. Reconstruction of the transverse mass from the $\tau$ jet and missing transverse energy requires a fully hadronic event, where the MET originates from the $\mathrm{H}^{ \pm}$decay. The events with leptonic $\mathrm{W}$ decays from the associated top were suppressed with a veto on isolated leptons.

The offline $\mathrm{E}_{\mathrm{T}}$ cut on the $\tau$ jet was taken to be $\mathrm{E}_{\mathrm{T}}^{\tau \text { jet }}>100 \mathrm{GeV}$. For an efficient isolation against the hadronic jets a small signal cone around the leading track, $\Delta \mathrm{R}=0.04$, and an isolation cone of $\Delta \mathrm{R}=0.4$ was selected. The $\tau$-jet isolation in the electromagnetic calorimeter was also applied. The $\tau$ helicity correlations were exploited requiring the leading track to carry at least $80 \%$ of the $\tau$ jet energy. The event reconstruction continued in events where at least three hadronic jets with $\mathrm{E}_{\mathrm{T}}>20 \mathrm{GeV}$ were found. A probabilistic secondary vertex algorithm with a discriminator cut was used for $b$ tagging [16]. The top-quark mass was reconstructed minimising the $\chi^{2}$ distribution build from the reconstructed and nominal top and $\mathrm{W}$ masses, $\chi^{2}=\left(\left(\mathrm{m}_{\mathrm{jj}}-\mathrm{m}_{\mathrm{W}}\right) / \sigma_{\mathrm{W}}\right)^{2}+\left(\left(\mathrm{m}_{\mathrm{jjj}}-\mathrm{m}_{\mathrm{top}}\right) / \sigma_{\mathrm{top}}\right)^{2}$, where $\mathrm{m}_{\mathrm{jj}}$ and $\mathrm{m}_{\mathrm{jjj}}$ are the invariant masses of all two- and three-jet combinations in the event and $\sigma_{\mathrm{W}}$ and $\sigma_{\text {top }}$ are the Gaussian widths of the reconstructed true $\mathrm{W}$ and top mass distributions. At least one of the jets was required to be assigned as a $b$ jet with $E_{T}>30 \mathrm{GeV}$. For further suppression of the $t \bar{t}$ background, the hadronic jets with $\mathrm{E}_{\mathrm{T}}>25 \mathrm{GeV}$ within $|\eta|<2.5$, remaining after top reconstruction, were vetoed. For the $\mathrm{t} \overline{\mathrm{t}}$, Wt and $\mathrm{W}+3$ jet backgrounds the configuration with large MET and large $\mathrm{E}_{\mathrm{T}}^{\tau \mathrm{jet}}$ can be reached only for strongly boosted $\mathrm{W}$. Therefore to suppress the background from events triggered with a fake $\tau$ from a hadronic jet recoiling against the genuine $\tau$ jet, a lower bound $\left(\mathrm{E}_{\mathrm{T}}^{\mathrm{H}}>50 \mathrm{GeV}\right)$ was set on the Higgs boson $\mathrm{p}_{\mathrm{T}}$ reconstructed from the $\tau$ jet and the missing transverse energy. The distribution of the transverse mass $\mathrm{m}_{\mathrm{T}}$, reconstructed from the $\tau$ jet and the missing $\mathrm{E}_{\mathrm{T}}$, is shown in Fig. 3 for $\mathrm{m}_{\mathrm{H}^{ \pm}}=170 \mathrm{GeV} / c^{2}$ and $\tan \beta=30$ for the signal and total background.

The probability of the background events to migrate to the signal area depends mainly on the precision of the jet energy and MET measurements. The main source of systematic uncertainty in the background measurement is the energy scale uncertainty. The systematic uncertainties due to $\tau$-jet identification, b-tagging, luminosity measurement, $t \bar{t}$ cross section and MonteCarlo statistics were also included. The total number of background events in the signal region, $\mathrm{m}_{\mathrm{T}}(\tau$ jet, MET $)>100 \mathrm{GeV}$, was found to be $1.7 \pm 1.0$ events, including the systematic uncertainties. Figure 1 shows the $5 \sigma$-discovery region in the $\mathrm{m}_{\mathrm{A}}-\tan \beta$ plane in the maximal mixing scenario with $\mu=200 \mathrm{GeV} / c^{2}$ for $30 \mathrm{fb}^{-1}$.

The $\mathrm{H}^{ \pm} \rightarrow \tau^{ \pm} v_{\tau}$ branching fractions used in Fig. 1 were calculated with FeynHiggs [18] including the decays to SUSY particles. The discovery potential including all SUSY corrections was investigated in Ref. [19]. The result on the $5 \sigma$-discovery potential for light and heavy charged 


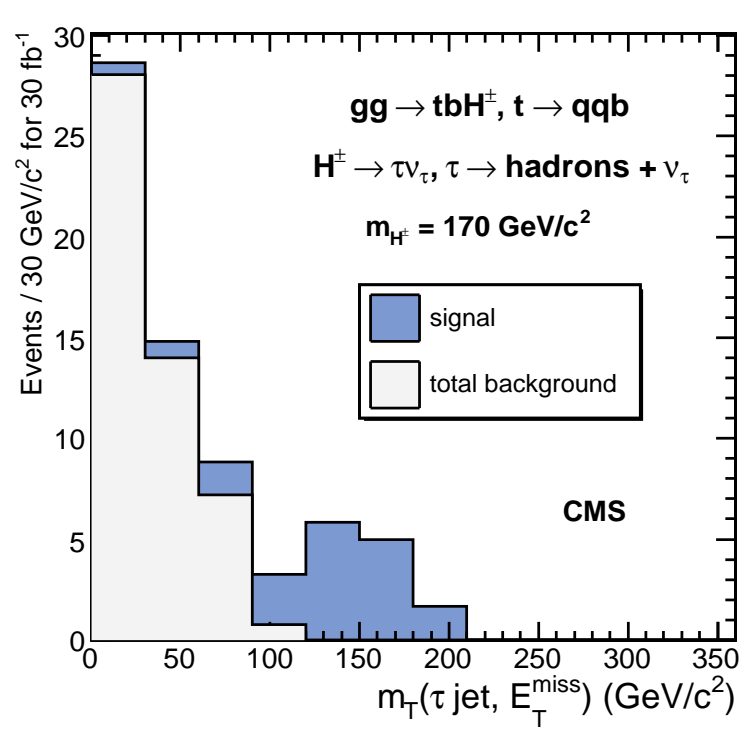

Figure 3: Transverse mass reconstructed from the $\tau$ jet and missing transverse energy for the $\mathrm{gg} \rightarrow \mathrm{tbH}^{ \pm}$, $\mathrm{t} \rightarrow \mathrm{bW}, \mathrm{W}^{\mp} \rightarrow \mathrm{jj}$ signal (dark histogram) with $\mathrm{m}_{\mathrm{H}^{ \pm}}$ $=170 \mathrm{GeV} / c^{2}, \tan \beta=30$ and for the total background (light histogram) for an integrated luminosity of $30 \mathrm{fb}^{-1}$.

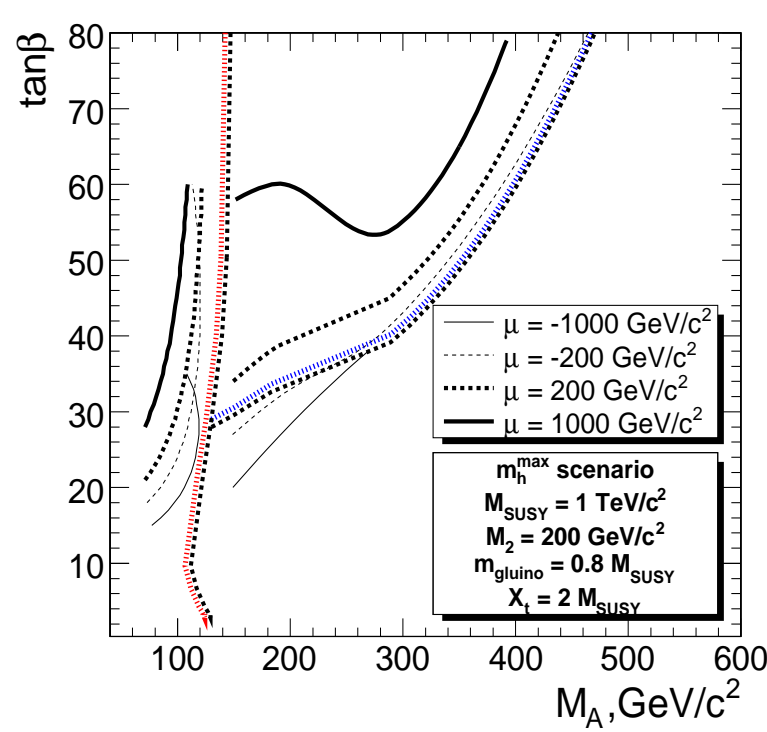

Figure 4: Discovery reach for the charged Higgs bosons with $30 \mathrm{fb}^{-1}$ in the $\mathrm{m}_{\mathrm{A}}, \tan \beta$ plane for $\mathrm{m}_{\mathrm{h}}^{\max }$ scenario for $\mu= \pm 200, \pm 1000 \mathrm{GeV}$ in comparison with the results from Ref. [9] (thickened dotted lines), obtained for $\mu=+200 \mathrm{GeV}$ and neglecting the SUSY corrections in the production process.

Higgs bosons is shown in Fig. 4 for $\mathrm{m}_{\mathrm{h}}^{\max }$ scenario and for $\mu= \pm 200, \pm 1000 \mathrm{GeV}$. Large negative $\mu$ values lead to largest reach while large positive $\mu$ values reduce the discovery regions significantly from those presented in Fig. 1. For the no-mixing scenario the SUSY correction effects are much less pronounced [19].

\section{New selection strategy and background considerations}

The forthcoming low luminosity LHC runs will be used to measure the SM backgrounds from data. Preparative studies with generated data are in progress for the $\mathrm{H}^{ \pm} \rightarrow \tau^{ \pm} v_{\tau}$ decay mode [20]. The $t \bar{t}$ and $\mathrm{W}+3 / 4$ jet events contribute to the background through genuine $\tau$ 's. Therefore the events passing the full selection can fall in the signal area $\left(\mathrm{m}_{\mathrm{T}}(\tau\right.$ jet, MET $\left.)>100 \mathrm{GeV} / \mathrm{c}^{2}\right)$ due to the uncertainty of the missing $\mathrm{E}_{\mathrm{T}}$ measurement. In the following a method will be discussed for the measurement of this background with muons in the muon+multi-jet events. The QCD multi-jet events are a large potential background through fake $\tau$ jets. To determine the contamination in the signal area the probability for a hadronic jet to pass the $\tau$-jet identification must be measured. The discovery strategy for the $\mathrm{H}^{ \pm} \rightarrow \tau^{ \pm} v_{\tau}$ channel is also revisited and improved methods are developed for the $\tau$ identification [21].

\subsection{Trigger updates}

The $\tau$ jet and missing $\mathrm{E}_{\mathrm{T}}$ at the HLT are used to trigger the events. The trigger threshold for the $\tau$ jet the Level- 1 is lowered from 96 to $80 \mathrm{GeV}$, better adapted to the low luminosity running. 
Isolation of the $\tau$ jet in ECAL is also included at the HLT. The tracker isolation is performed at two levels, which makes possible to lower the track $\mathrm{p}_{\mathrm{T}}$ threshold to $1 \mathrm{GeV} / \mathrm{c}$ in the isolation cone.

\subsection{Identification of the $\tau$-jet from $\mathrm{H}^{ \pm} \rightarrow \tau^{ \pm} v_{\tau}$}

The new development of $\tau$ identification is optimised to suppress the hadronic jets from QCD multi-jet events as efficiently as possible [21]. The genuine $\tau$ jets coming from the $\mathrm{W} \rightarrow \tau v$ decays in the $t \bar{t}$ and $\mathrm{W}+3 / 4$ jet backgrounds have lower $\mathrm{E}_{\mathrm{T}}$ values and are therefore suppressed with $\mathrm{E}_{\mathrm{T}}$ cut but a strong suppression of these $\tau$ jets is obtained from the requirement of the leading track carrying most of the $\tau$-jet energy, taking advantage of the helicity correlations. The jets reconstructed in the calorimeter and corrected with energy corrections based on MC studies were used as $\tau$ candidates. The $\eta$ range for the $\tau$-jet candidates was reduced to $|\eta|<2$ to exploit the kinematic difference of the signal $\tau$ jets and the QCD jets. Isolation was performed in the tracker and in ECAL. The new track reconstruction developments of CMS allow the track $\mathrm{p}_{\mathrm{T}}$ threshold to be lowered down to $\sim 0.5 \mathrm{GeV} / \mathrm{c}$, which significantly improves the isolation power. Cuts on the track/HCAL matching were introduced to reject neutral hadrons and electrons in the $\tau$ candidate jet. The selection was optimised separately for one- and three-prong $\tau$ jets. For the one-prong selection the helicity correlations in the $\tau$ decay were exploited requiring that the leading track carries more than $80 \%$ of the $\tau$ jet energy. For the three-prong selection, the final states without $\pi^{0}$ 's, presenting $2 / 3$ of the $\tau \rightarrow \mathrm{a}_{1} v_{\tau}$ decays, were selected requiring matching between the tracker momentum and the energy of the jet. For the $\tau$ jets without $\pi^{0}$ 's the helicity correlations can be exploited requiring that the momentum sum of the two same-sign pions carry more than $75 \%$ of the $\tau$ jet energy for the three-prong selection, as has been shown in Ref. [22]. Furthermore the $\tau$ mass requirement can be used efficiently in the selected decay mode without $\pi^{0}$ 's, reconstructing the invariant mass from the three tracks. Further background reduction was obtained reconstructing the $\tau$ decay vertex and setting a cut on the significance on the $\tau$ flight path. The sum of track charges was required to be \pm 1 . Preliminary result from the new $\tau$ selection yields a factor of $\sim 10$ improvement on the ratio of signal $\tau$ jets to the fake $\tau$ jets from QCD processes. The three-prong final states were found to increase significantly the signal efficiency without excessive increase of the background from hadronic jets.

\subsection{Event selection}

The remaining event selection was performed with the lines discussed in Section 4. Events with an identified electron or muon were rejected. For more efficient leptonic veto, the $\mathrm{p}_{\mathrm{T}}$ thresholds for electrons and muons were set to lower values ( $8 \mathrm{GeV} / c$ for electrons and $4 \mathrm{GeV} / c$ muons), and no isolation requirements were applied on the muons. A kinematic fit was introduced to reconstruct the $\mathrm{W}$ and top masses, varying the jet energies within the measured uncertainties and taking the nominal $\mathrm{W}$ and top masses as constraints in the fit. The $\mathrm{b}$ jet for the top mass was selected as the one with maximum discriminator value and with $\mathrm{E}_{\mathrm{T}}>30 \mathrm{GeV}$, to select the more energetic $\mathrm{b}$ jet from top decay and to avoid the $\mathrm{b}$ jet from the production process, emitted in more forward regions. The quark jets assigned to the $\mathrm{W}$ were selected from the remaining jets with $\mathrm{E}_{\mathrm{T}}>20 \mathrm{GeV}$. The kinematic fit was performed to all jet combinations, and the combination with the smallest $\chi^{2}$ was selected. Contrary to the study of Ref. [17], no central jet veto was applied. 


\subsection{Plan for $\mathrm{t} \bar{t}$ and $W+3 / 4 j e t$ backgrounds}

The $t \bar{t}$ and $\mathrm{W}+3 / 4$ jet events remaining after all selection cuts fall into the "background" area $\mathrm{m}_{\mathrm{T}}(\tau$ jet, MET $)<\mathrm{m}_{\mathrm{W}}$, if the missing transverse energy is correctly measured. The background due to MET resolution is planned to be measured with the muon+multi-jet events, replacing the muon with a $\tau$ jet and performing the full event selection.

Preliminary study was performed with $t \bar{t}$ and $\mathrm{W}+3 / 4$ jet events in the CMS detector to measure the background due to MET resolution [20]. Muons from the global muon reconstruction were used for searching one isolated muon with $\mathrm{p}_{\mathrm{T}}>100 \mathrm{GeV} / \mathrm{c}^{2}$ from $\mathrm{W} \rightarrow \mu v$. Events with other reconstructed muons with $\mathrm{p}_{\mathrm{T}}^{\mu}>4 \mathrm{GeV} / \mathrm{c}$ and events with identified electrons with with $\mathrm{p}_{\mathrm{T}}^{\mathrm{e}}>10 \mathrm{GeV} / \mathrm{c}$ were rejected. The event selection was continued demanding a large missing $\mathrm{E}_{\mathrm{T}}, \mathrm{MET}>100 \mathrm{GeV}$, and three hadronic jets with $\mathrm{E}_{\mathrm{T}}^{\mathrm{jet}}>20 \mathrm{GeV}$. The muon replacement with $\tau$ jet was not yet performed, but instead the data selected was divided into samples of $t \bar{t}$ and $W+3 / 4$ jet events. The $\mathrm{t} \overline{\mathrm{t}}$ events were selected requiring two tagged $\mathrm{b}$ jets and hadronic $\mathrm{W}$ and top masses reconstructed with the kinematic fit described in Section 6.3. Most of the events passing all selection cuts and falling at large $\mathrm{m}_{\mathrm{T}}(\mu, \mathrm{MET})$ values were found to contain a hadronic $\tau$ decay, and thus an additional neutrino, from the associated top: $\mathrm{t} \rightarrow \mathrm{bW}, \mathrm{W} \rightarrow \tau \nu$. The background events falling close to $\mathrm{m}_{\mathrm{T}}(\mu, \mathrm{MET}) \sim 100 \mathrm{GeV} / \mathrm{c}^{2}$ were found to be more often fully hadronic containing the $\mathrm{W} \rightarrow \mathrm{ud}$ and $\mathrm{W} \rightarrow$ cs decays from the associated $\mathrm{W}$.

\subsection{Importance of hadronic purity for missing $E_{T}$ measurement}

Separation of signal and background in the $\mathrm{m}_{\mathrm{T}}(\mu, \mathrm{MET})\left(\right.$ or $\mathrm{m}_{\mathrm{T}}(\tau$ jet, MET) ) distribution is possible when the only source of MET is the neutrino produced in association of the observed muon (or $\tau$ jet). The leptonic decays of the $\mathrm{W}$ from the associated top produce an additional neutrino and can therefore alter the magnitude and direction of the the MET measurement. The W decays to electrons, muons and to leptonically decaying $\tau$ 's can be efficiently suppressed with the lepton veto methods. As was shown in Section 6.4, the hadronic $\tau$ decays of the $\mathrm{W}$ remain and can lead to signal-like events with large $\mathrm{m}_{\mathrm{T}}(\tau$ jet, MET). These associated $\tau$ jets are difficult to identify due to the low $\mathrm{E}_{\mathrm{T}}$ values. The Particle Flow (PF) method can improve the identification efficiency significantly but it may be difficult to obtain a clean MET measurement with the veto methods alone. Another possibility to suppress the leptonic associated $\mathrm{W}$ decays is a precise hadronic $\mathrm{W}$ and top mass reconstruction and tight mass window cuts on the reconstructed masses.

\subsection{Plan for the measurement of the QCD multi-jet background}

The QCD multi-jet events can fall to the signal area due to mis-identification of a hadronic jet as a $\tau$ jet and due to uncertainties of the MET measurement. The background at large $\mathrm{m}_{\mathrm{T}}$ values $(\gtrsim 100 \mathrm{GeV}$ ) can be estimated in the hadronic multi-jet events, determining the $\tau$ misidentification efficiency from other processes. In a preliminary study events with at least one hadronic jet with $\mathrm{E}_{\mathrm{T}}>100 \mathrm{GeV}$ were first selected in the QCD multi-jet samples with $80<$ $\hat{\mathrm{p}}_{\mathrm{T}}<120 \mathrm{GeV} / \mathrm{c}, 120<\hat{\mathrm{p}}_{\mathrm{T}}<170 \mathrm{GeV} / \mathrm{c}$ and $170<\hat{\mathrm{p}}_{\mathrm{T}}<230 \mathrm{GeV} / \mathrm{c}$. One of these jets, selected randomly, was taken as the $\tau$ candidate. The event selection was continued demanding a large missing $\mathrm{E}_{\mathrm{T}}(\mathrm{MET}>100 \mathrm{GeV}$ ), searching for the best b-tagged jet and reconstructing the $\mathrm{W}$ and top masses. The transverse mass $\mathrm{m}_{\mathrm{T}}(\tau$ candidate, MET $)$ was reconstructed from the $\tau$-jet candidate 
and the MET. Contamination observed in the signal area was found to originate from badly measured jets leading to large MET values. Correction methods for MET measurement are in progress to suppress this background.

Standard method to measure the fake $\tau$ probability is to use the balanced $\gamma+$ jet or Z+jet, $\mathrm{Z} \rightarrow \mu \mu$ events. Preliminary estimates indicate that for a reasonable statistical accuracy of the measurement integrated luminosities of the order of $10 \mathrm{fb}^{-1}$ are required due to the large $\mathrm{E}_{\mathrm{T}}$ scale needed. Furthermore the flavour content of the jet in the $\gamma+$ jet events differs from that of the jets produced in the QCD multi-jet events. Therefore a more convenient way to measure the fake $\tau$ probability for $\mathrm{H}^{ \pm}$searches is to use the QCD di-jet events, taken with pre-scaled jet triggers and having large production rate.

\section{Conclusions}

The $\mathrm{H}^{ \pm} \rightarrow \tau \nu_{\tau}$ decay channel with hadronic $\tau$ decays plays a crucial rule in the charged Higgs boson searches. For $\mathrm{m}_{\mathrm{H}^{ \pm}}<\mathrm{m}_{\text {top }}$ searches in the $\mathrm{t} \overline{\mathrm{t}}$ events with leptonic triggers from the decay of one of the top quarks were discussed. The SUSY correction in the production are unfavourable and reduce the discovery region in particular at small $\tan \beta$ values. Searches for heavy charged Higgs bosons were discussed with the $\mathrm{H}^{ \pm} \rightarrow \tau v_{\tau}$ decay channel in the associated production with a top quark with fully hadronic final state. This channel yields a discovery at large $\tan \beta(\gtrsim 30$ around $\mathrm{m}_{\mathrm{A}}=200 \mathrm{GeV} / \mathrm{c}^{2}$ ) for $30 \mathrm{fb}^{-1}$ with $\mu=200 \mathrm{GeV} / \mathrm{c}^{2}$. SUSY corrections on the production can reduce the discovery region significantly in the maximal mixing scenario for large positive $\mu$ values. Some enhancement of the discovery region can be expected for large negative $\mu$ values. For the searches of the charged Higgs bosons the dominating $\mathrm{H}^{ \pm} \rightarrow$ tb decay mode with leptonic triggers were also discussed. Visible signal may not be obtained with this channel for $30 \mathrm{fb}^{-1}$ in the expected parameter space. Some enlargement of the overall discovery region could be expected from fully hadronic final states for the light charged Higgs bosons in the $\bar{t} \bar{t}$ events and from the $\mathrm{H}^{ \pm} \rightarrow \tau v_{\tau}, \tau \rightarrow \mu \mathrm{e} v v_{\tau}$ decay channel, not yet investigated in CMS.

New developments for the analysis methods were discussed. Preliminary results indicate that the hadronic QCD multi-jet background can be suppressed more efficiently with the newly developed $\tau$ identification method. The method is based on efficient isolation in particular with the tracker and on separate optimisation of the cuts for the one- and three-prong $\tau$ jets. Possibility to exploit the helicity correlations plays a central role: the requirement of the leading track to carry at least $80 \%$ of the $\tau$-jet energy suppresses efficiently the $t \bar{t}$ and $\mathrm{W}+3 / 4$ jet backgrounds and gives a large further suppression factor against the hadronic jets.

Plans for the measurement of the SM backgrounds in the signal area from the early LHC data was discussed. The $t \bar{t}$ and $\mathrm{W}+3 / 4$ jet components can be measured with the muon+multi-jet events, replacing the reconstructed muon with a generated $\tau$ and performing the event selection. Unidentified hadronic $\tau$ decays from the associated top decay were found to be an important background source, through contamination of the MET measurement. For the QCD multi-jet background the main issue is the measurement of the probability for a hadronic jet to pass the $\tau$-jet identification. This measurement may be performed with events passing the di-jet triggers already with the luminosities of $\sim 1 \mathrm{fb}^{-1}$. The second background component in the QCD multi-jet events, the tail of the MET measurement due to mis-measured jets, will be suppressed with MET correction methods. 


\section{References}

[1] Kenzo Inoue, Akira Kakuto, Hiromasa Komatsu, and Seiichiro Takeshita. Aspects of Grand Unified Models with Softly Broken Supersymmetry. Prog. Theor. Phys., 68:927, 1982.

[2] Savas Dimopoulos and Howard Georgi. Softly Broken Supersymmetry and SU(5). Nucl. Phys., B193:150, 1981.

[3] N. Sakai. Naturalness in Supersymmetric Guts. Zeit. Phys., C11:153, 1981.

[4] The LEP Working Group for Higgs Boson Searhes. LHWG-Note 2004-01.

[5] D. P. Roy. The hadronic tau decay signature of a heavy charged Higgs boson at LHC. Phys. Lett., B459:607-614, 1999.

[6] D. P. Roy. Looking for the charged Higgs boson. Mod. Phys. Lett., A19:1813-1828, 2004.

[7] S. Jadach, Z. Was, R. Decker, and J. Kuhn. Comp. Phys. Commun., 76:361, 1993.

[8] M Baarmand, M Hashemi, and A Nikitenko. Light Charged Higgs Discovey Potential of CMS in the $\mathrm{H}^{ \pm} \rightarrow \tau \nu$ Decay with Single Lepton Trigger. CMS Note 2006/056.

[9] The CMS Collaboration. CMS Physics TDR:Volume II, Physics Performance. CERN/LHCC 2006-021, 2006.

[10] Torbjorn Sjostrand et al. High-energy-physics event generation with PYTHIA 6.1. Comput. Phys. Commun., 135:238-259, 2001.

[11] S.R Slabospitsky and L. Sonnenschein. TopRex 3.25 - A specialized event generator for use with PYTHIA. Comput. Phys. Commun., 148:87-102, 2002.

[12] J. Alwall, P. Demin, S. de Visscher, R. Frederix, M. Herquet, F. Maltoni, T. Plehn, and T Rainwater, D.L. anf Stelzer. MadGraph/MadEvent v4: The New Web Generation. JHEP, 0709:028, 2007.

[13] S. Lowette, J. D'Hondt, and P. Vanlaer. Charged MSSM Higgs Boson Observability in the $\mathrm{H}^{ \pm} \rightarrow$ tb. CMS Note 2006/109.

[14] Tilman Plehn. Charged Higgs boson production in bottom gluon fusion. Phys. Rev., D67:014018, 2003.

[15] A Pukhov, E Boos, M Dubinin, V Edneral, V Ilyin, D Kovalenko, A Kryukov, V Savrin, S Shichanin, and A Semenov. CompHEP - a package for evaluation of Feynman diagrams and integration over multi-particle phase space. INP-MSU 98-41/542.

[16] C Weiser. A Combined Secondary Vertex Based B-Tagging Algorithm in CMS. CMS Note 2006/014.

[17] R Kinnunen. Search for the heavy charged MSSM Higgs bosons with the $\mathrm{H}^{ \pm} \rightarrow \tau^{ \pm} v_{\tau}$ decay mode in fully hadronic final state, revised version. CMS Note 2006/100.

[18] Sven Heinemeyer, Georg Weiglein, and Wolfgang Hollik. Comput.Phys.Commun., 124:76-89, 2000.

[19] M. Hashemi, S. Heinemeyer, R. Kinnunen, A. Nikitenko, and G. Weiglein. Charged Higgs Bosons in the MSSM at CMS: Discovery Reach and Parameter Dependence. arXiv:0804.1228, 2008.

[20] CMS Collaboration. Measurement of SM backgrounds for heavy charged Higgs bosons with $\mathrm{H}^{ \pm} \rightarrow \tau^{ \pm} v_{\tau}$. Physics Analysis Summary in preparation.

[21] CMS Collaboration. Identification of $\tau$ jets from $\mathrm{H}^{ \pm} \rightarrow \tau^{ \pm} v_{\tau}$. Physics Analysis Summary in preparation.

[22] Monoranjan Guchait, Ritva Kinnunen, and D. P. Roy. Signature of heavy charged Higgs boson at LHC in the 1 and 3 prong hadronic tau decay channels. Eur. Phys. J., C52:665-672, 2007. 